\title{
DÜBLIN
}

Technological University Dublin

ARROW@TU Dublin

2009-01-01

\section{Poisson Structures of Equations associated with groups of diffeomorphisms}

Rossen Ivanov

Technological University Dublin, rossen.ivanov@tudublin.ie

Follow this and additional works at: https://arrow.tudublin.ie/scschmatcon

Part of the Geometry and Topology Commons, Non-linear Dynamics Commons, and the Partial Differential Equations Commons

\section{Recommended Citation}

Ivanov, R. (2009). Poisson Structures of Equations associated with groups of diffeomorphisms. World Scientific, pg 99 - 108. doi:10.21427/nm8q-hn16

This Conference Paper is brought to you for free and open access by the School of Mathematics at ARROW@TU Dublin. It has been accepted for inclusion in Conference papers by an authorized administrator of ARROW@TU Dublin. For more information, please contact arrow.admin@tudublin.ie, aisling.coyne@tudublin.ie, gerard.connolly@tudublin.ie. Funder: INTAS grant No 05-1000008-7883

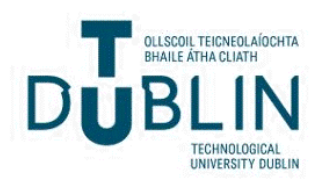




\title{
POISSON STRUCTURES OF EQUATIONS ASSOCIATED WITH GROUPS OF DIFFEOMORPHISMS
}

\author{
R. I. IVANOV $\ddagger$ \\ School of Mathematical Sciences, Dublin Institute of Technology, \\ Kevin Street, Dublin 8, Ireland \\ $\ddagger$ E-mail: rivanov@dit.ie
}

A class of equations describing the geodesic flow for a right-invariant metric on the group of diffeomorphisms of $\mathbb{R}^{n}$ is reviewed from the viewpoint of their Lie-Poisson structures. A subclass of these equations is analogous to the Euler equations in hydrodynamics (for $n=3$ ), preserving the volume element of the domain of fluid flow. An example in $n=1$ dimension is the Camassa-Holm equation, which is a geodesic flow equation on the group of diffeomorphisms, preserving the $H^{1}$ metric.

Keywords: Lie group, Virasoro group, group of diffeomorphisms, Lie-Poisson bracket, vector fields

\section{Camassa-Holm equation}

The Camassa-Holm $(\mathrm{CH})$ equation can be considered as a member of the family of EPDiff equations, that is, Euler-Poincaré equations, associated with the diffeomorphism group in $n$-dimensions. ${ }^{18}$ Let us consider first the $\mathrm{CH}$ equation in the form

$$
q_{t}+2 u_{x} q+u q_{x}=0, \quad q=u-u_{x x}+\omega,
$$

with $\omega$ an arbitrary parameter. The traveling wave solutions of (1) are smooth solitons ${ }^{5}$ if $\omega>0$ and peaked solitons (peakons) if $\omega=0.4,13,14,24,28$

$\mathrm{CH}$ is a bi-hamiltonian equation, i.e. it admits two compatible Hamiltonian structures ${ }^{4,15} J_{1}=-(q \partial+\partial q), J_{2}=-\left(\partial-\partial^{3}\right)$ :

$$
\begin{aligned}
q_{t} & =J_{2} \frac{\delta H_{2}[q]}{\delta q}=J_{1} \frac{\delta H_{1}[q]}{\delta q}, \\
H_{1} & =\frac{1}{2} \int q u \mathrm{~d} x, \\
H_{2} & =\frac{1}{2} \int\left(u^{3}+u u_{x}^{2}+2 \omega u^{2}\right) \mathrm{d} x .
\end{aligned}
$$


If $\omega \neq 0$ the invariance group of the Hamiltonian is the Virasoro group, Vir $=\operatorname{Diff}\left(\mathbb{S}^{1}\right) \times \mathbb{R}$ and the central extension of the corresponding Virasoro algebra is proportional to $\omega \cdot^{10-12,19,25,30}$ Thus $\mathrm{CH}$ has various conformal properties. ${ }^{21}$ It is also completely integrable, possesses bi-Hamiltonian form and infinite sequence of conservation laws. $4,8,9,22,32$

The soliton solution has the form

$$
q(x, t)=\int_{0}^{\infty} \delta(x-X(\xi, t)) P(\xi, t) d \xi,
$$

where $X(\xi, t)$ and $P(\xi, t)$ are quantities well defined in terms of the scattering data ${ }^{7,8,10}\left(q(x, 0)>0\right.$ is assumed, otherwise wave breaking occurs $\left.{ }^{6}\right)$. From (5) one can easily compute $u=\left(1-\partial^{2}\right)^{-1}(q-\omega)$,

$$
u(x, t)=\frac{1}{2} \int_{0}^{\infty} e^{-|x-X(\xi, t)|} P(\xi, t) d \xi-\omega .
$$

Substitution of (5) and (6) into the equation (1) and using the fact that

$$
f(x) \delta^{\prime}\left(x-x_{0}\right)=f\left(x_{0}\right) \delta^{\prime}\left(x-x_{0}\right)-f^{\prime}\left(x_{0}\right) \delta\left(x-x_{0}\right)
$$

we derive a system of integral equations for $X$ and $P$ :

$$
\begin{array}{r}
X_{t}(\xi, t)=\int G(X(\xi, t)-X(\underline{\xi}, t)) P(\underline{\xi}, t) \mathrm{d} \underline{\xi}-\omega, \\
P_{t}(\xi, t)=-\int G^{\prime}(X(\xi, t)-X(\underline{\xi}, t)) P(\xi, t) P(\underline{\xi}, t) \mathrm{d} \underline{\xi},
\end{array}
$$

where $G(x) \equiv \frac{1}{2} e^{-|x|}$. From (5) and (6) the Hamiltonian $H_{1}$ can be expressed as

$H_{1}(X, P)=\frac{1}{2} \int G\left(X\left(\xi_{1}, t\right)-X\left(\xi_{2}, t\right)\right) P\left(\xi_{1}, t\right) P\left(\xi_{2}, t\right) \mathrm{d} \xi_{1} \mathrm{~d} \xi_{2}-\omega \int P(\xi, t) \mathrm{d} \xi$ and the equations (7) and (8) as

$$
X_{t}(\xi, t)=\frac{\delta H}{\delta P(\xi, t)}, \quad P_{t}(\xi, t)=-\frac{\delta H}{\delta X(\xi, t)},
$$

i.e. these equations are Hamiltonian, with respect to the canonical Poisson bracket

$$
\{A, B\}_{c}=\int\left(\frac{\delta A}{\delta X(\xi, t)} \frac{\delta B}{\delta P(\xi, t)}-\frac{\delta B}{\delta X(\xi, t)} \frac{\delta A}{\delta P(\xi, t)}\right) \mathrm{d} \xi .
$$

and the canonical variables are $X(\xi, t), P(\xi, t)$ :

$$
\begin{array}{r}
\left\{X\left(\xi_{1}, t\right), P\left(\xi_{2}, t\right)\right\}_{c}=\delta\left(\xi_{1}-\xi_{2}\right), \\
\left\{P\left(\xi_{1}, t\right), P\left(\xi_{2}, t\right)\right\}_{c}=\left\{X\left(\xi_{1}, t\right), X\left(\xi_{2}, t\right)\right\}_{c}=0 .
\end{array}
$$


Now we can show that (5) is a momentum map that produces the Poisson brackets, given by the Hamiltonian operator $J_{1}$. To do this we will use the canonical Poisson brackets (11), (12) to compute $\left\{q\left(x_{1}\right), q\left(x_{2}\right)\right\}_{c}$.

Indeed

$$
\begin{aligned}
& \left\{q\left(x_{1}, t\right), q\left(x_{2}, t\right)\right\}_{c}= \\
& \left\{\int_{0}^{\infty} \delta\left(x_{1}-X\left(\xi_{1}, t\right)\right) P\left(\xi_{1}, t\right) d \xi_{1}, \quad \int_{0}^{\infty} \delta\left(x_{2}-X\left(\xi_{2}, t\right)\right) P\left(\xi_{2}, t\right) d \xi_{2}\right\}_{c}= \\
- & \int_{0}^{\infty} \int_{0}^{\infty}\left\{X\left(\xi_{1}, t\right), P\left(\xi_{2}, t\right)\right\}_{c} \delta^{\prime}\left(x_{1}-X\left(\xi_{1}, t\right)\right) P\left(\xi_{1}, t\right) \delta\left(x_{2}-X\left(\xi_{2}, t\right)\right) d \xi_{1} d \xi_{2} \\
- & \int_{0}^{\infty} \int_{0}^{\infty}\left\{P\left(\xi_{1}, t\right), X\left(\xi_{2}, t\right)\right\}_{c} \delta\left(x_{1}-X\left(\xi_{1}, t\right)\right) \delta^{\prime}\left(x_{2}-X\left(\xi_{2}, t\right)\right) P\left(\xi_{2}, t\right) d \xi_{1} d \xi_{2} \\
=- & \delta^{\prime}\left(x_{1}-x_{2}\right) \int_{0}^{\infty} P\left(\xi_{2}, t\right) \delta\left(x_{2}-X\left(\xi_{2}, t\right)\right) d \xi_{2}+ \\
=- & q\left(x_{2}, t\right) \delta^{\prime}\left(x_{1}-x_{2}\right)+q\left(x_{1}, t\right) \delta^{\prime}\left(x_{2}-x_{1}\right) \int_{0}^{\infty} P\left(\xi_{1}, t\right) \delta\left(x_{1}-X\left(\xi_{1}, t\right)\right) d \xi_{1} \\
= & -\left(q\left(x_{1}, t\right) \frac{\partial}{\partial x_{1}}+\frac{\partial}{\partial x_{1}} q\left(x_{1}, t\right)\right) \delta\left(x_{1}-x_{2}\right)=J_{1}\left(x_{1}\right) \delta\left(x_{1}-x_{2}\right) .
\end{aligned}
$$

Now it is straightforward to check, using (2), that (1) can be written in a Hamiltonian form as

$$
q_{t}=\left\{q, H_{1}\right\}_{c}
$$

with the Poisson bracket, generated by $J_{1}$ :

$$
\begin{aligned}
\{A, B\}_{c} & =\int \frac{\delta A}{\delta q(x)} J_{1}(x) \frac{\delta B}{\delta q(x)} \mathrm{d} x \\
& =-\int q(x)\left(\frac{\delta A}{\delta q(x)} \frac{\partial}{\partial x} \frac{\delta B}{\delta q(x)}-\frac{\delta B}{\delta q(x)} \frac{\partial}{\partial x} \frac{\delta A}{\delta q(x)}\right) \mathrm{d} x
\end{aligned}
$$

A singular momentum map of type (5) is used $^{18}$ for the construction of peakon, filament and sheet singular solutions for higher dimensional EPDiff equations.

The parallel with the geometric interpretation of the integrable $\mathrm{SO}(3)$ top can be made explicit by a discretization of $\mathrm{CH}$ equation based on Fourier modes expansion. ${ }^{23}$ Since the Virasoro algebra is an infinite-dimensional algebra, the obtained equation represents an 'integrable top' with infinitely many momentum components.

If we compare (6) and (7) we have

$$
X_{t}(\xi, t)=u(X(\xi, t), t)
$$


i.e. $X(\xi, t)$ is explicitly the diffeomorphism related to the geodesic curve, ${ }^{10,27,30}$ i.e. $X(x, t)$ is an one-parameter curve of diffeomorphisms of $\mathbb{R}$ (or, with periodic boundary conditions, of the circle $\mathbb{S}^{1}$ ), depending on a parameter $t$ and associated with a right-invariant metric given by the Hamiltonian $H_{1}$.

For the peakon solutions $(\omega=0)$ the dependence on the scattering data is also known. For completeness and comparison we mention the analogous results for this case. The $N$-peakon solution has the form ${ }^{2,4}$

$$
u(x, t)=\frac{1}{2} \sum_{i=1}^{N} p_{i}(t) \exp \left(-\left|x-x_{i}(t)\right|\right),
$$

provided $p_{i}$ and $x_{i}$ evolve according to the following system of ordinary differential equations:

$$
\dot{x}_{i}=\frac{\partial H}{\partial p_{i}}, \quad \dot{p}_{i}=-\frac{\partial H}{\partial x_{i}},
$$

where the Hamiltonian is $H=\frac{1}{4} \sum_{i, j=1}^{N} p_{i} p_{j} \exp \left(-\left|x_{i}-x_{j}\right|\right)$. Now one can see immediately the analogy between $X(\xi, t)$ and $x_{i}(t) ; P(\xi, t)$ and $p_{i}(t)$ due to the fact that the $N$-soliton solution with the limit $\omega \rightarrow 0$ converges to the $N$-peakon solution. ${ }^{3}$

\section{2. $n$-dimensional EPDiff equations}

Let us consider motion in $\mathbb{R}^{n}$ with a velocity field $\mathbf{u}(\mathbf{x}, t): \mathbb{R}^{n} \times \mathbb{R} \rightarrow \mathbb{R}^{n}$ and define a momentum variable $\mathbf{m}=Q \mathbf{u}$ for some (inertia) operator $Q$ (for CH generalizations $Q$ is the Helmholtz operator $Q=1-\partial_{i} \partial_{i}=1-\Delta$, where $\left.\partial_{i}=\frac{\partial}{\partial x^{i}}\right)$. The kinetic energy defines a Lagrangian

$$
L[\mathbf{u}]=\frac{1}{2} \int \mathbf{m} \cdot \mathbf{u} \mathrm{d}^{n} \mathbf{x} .
$$

Since the velocity $\mathbf{u}=u^{i} \partial_{i}$ is a vector field, $\mathbf{m}=m_{i} \mathrm{~d} x^{i} \otimes \mathrm{d}^{n} \mathbf{x}$ is a $n+1$ form density, we have a natural bilinear form

$$
\langle\mathbf{m}, \mathbf{u}\rangle=\int \mathbf{m} \cdot \mathbf{u} \mathrm{d}^{n} \mathbf{x} .
$$

The The Euler-Poincaré equation for the geodesic motion is ${ }^{18,19}$

$$
\frac{d}{d t} \frac{\delta L}{\delta \mathbf{u}}+\operatorname{ad}_{\mathbf{u}}^{*} \frac{\delta L}{\delta \mathbf{u}}=0, \quad \mathbf{u}=G * \mathbf{m},
$$

where $G$ is the Green function for the operator $Q$. The corresponding Hamiltonian is

$$
H[\mathbf{m}]=\langle\mathbf{m}, \mathbf{u}\rangle-L[\mathbf{u}]=\frac{1}{2} \int \mathbf{m} \cdot G * \mathbf{m} \mathrm{d}^{n} \mathbf{x},
$$


and the equation in Hamiltonian form $\left(\mathbf{u}=\frac{\delta H}{\delta \mathbf{m}}\right)$ is

$$
\frac{\partial \mathbf{m}}{\partial t}=-\operatorname{ad}_{\frac{\delta H}{\delta \mathbf{m}}}^{*} \mathbf{m}
$$

The left Lie algebra of vector fields is $[\mathbf{u}, \mathbf{v}]=-\left(u^{k}\left(\partial_{k} v^{p}\right)-v^{k}\left(\partial_{k} u^{p}\right)\right) \partial_{p}$. For an arbitrary vector field $\mathbf{v}$ one can write ${ }^{19}$

$$
\begin{aligned}
\left\langle\operatorname{ad}_{\mathbf{u}}^{*} \mathbf{m}, \mathbf{v}\right\rangle & =\left\langle\mathbf{m}, \operatorname{ad}_{\mathbf{u}} \mathbf{v}\right\rangle=\langle\mathbf{m},[\mathbf{u}, \mathbf{v}]\rangle \\
& =-\left\langle m_{l} \mathrm{~d} x^{l} \otimes \mathrm{d}^{n} \mathbf{x},\left(u^{k}\left(\partial_{k} v^{p}\right)-v^{k}\left(\partial_{k} u^{p}\right)\right) \partial_{p}\right\rangle \\
& =-\int m_{p}\left(u^{k}\left(\partial_{k} v^{p}\right)-v^{k}\left(\partial_{k} u^{p}\right)\right) \mathrm{d}^{n} \mathbf{x} \\
= & -\int\left(\partial_{k}\left(m_{p} u^{k} v^{p}\right)-\left(\partial_{k} m_{p}\right) u^{k} v^{p}-m_{p} v^{p}\left(\partial_{k} u^{k}\right)-m_{p} v^{k}\left(\partial_{k} u^{p}\right)\right) \mathrm{d}^{n} \mathbf{x} \\
& =\int v^{p}\left(u^{k}\left(\partial_{k} m_{p}\right)+m_{p}\left(\partial_{k} u^{k}\right)+m_{k}\left(\partial_{p} u^{k}\right)\right) \mathrm{d}^{n} \mathbf{x} \\
& =\left\langle\left((\mathbf{u} \cdot \nabla) m_{p}+\mathbf{m} \cdot \partial_{p} \mathbf{u}+m_{p} \operatorname{div} \mathbf{u}\right) \mathrm{d} x^{p} \otimes \mathrm{d}^{n} \mathbf{x}, \mathbf{v}\right\rangle,
\end{aligned}
$$

and therefore (21) has the form

$$
\frac{\partial m_{p}}{\partial t}+(\mathbf{u} \cdot \nabla) m_{p}+\mathbf{m} \cdot \partial_{p} \mathbf{u}+m_{p} \operatorname{div} \mathbf{u}=0 .
$$

Let us now define an one-parametric group of diffeomorphisms of $\mathbb{R}^{n}$, with elements that satisfy

$$
\frac{\partial \mathbf{X}(\mathbf{x}, t)}{\partial t}=\mathbf{u}(\mathbf{X}(\mathbf{x}, t), t), \quad \mathbf{X}(\mathbf{x}, 0)=\mathbf{x} .
$$

Due to the invariance of the Hamiltonian under the action of the group there is a momentum conservation law:

$$
m_{i}(\mathbf{X}(\mathbf{x}, t), t) \partial_{j} X^{i}(\mathbf{x}, t) \operatorname{det}\left(\frac{\partial \mathbf{X}}{\partial \mathbf{x}}\right)=m_{j}(x, 0),
$$

where $\left(\frac{\partial \mathbf{X}}{\partial \mathbf{x}}\right)_{i j}=\frac{\partial X^{i}}{\partial x^{j}}$ is the Jacobian matrix.

The Lie-Poisson bracket is

$$
\begin{aligned}
\{A, B\}(\mathbf{m}) & =\left\langle\mathbf{m},\left[\frac{\delta A}{\delta \mathbf{m}}, \frac{\delta B}{\delta \mathbf{m}}\right]\right\rangle \\
& =-\int m_{i}\left(\frac{\delta A}{\delta m_{k}} \partial_{k} \frac{\delta B}{\delta m_{i}}-\frac{\delta B}{\delta m_{k}} \partial_{k} \frac{\delta A}{\delta m_{i}}\right) \mathrm{d}^{n} \mathbf{x} .
\end{aligned}
$$

When $n=1$ clearly (25) gives (13) and the algebra, associated with the bracket is the algebra of vector fields on the circle. This algebra admits a generalization with a central extension, which is the famous Virasoro algebra. ${ }^{10-12,19,25,30}$ In two dimensions, $n=2$, the algebra, associated with the bracket is the algebra of vector fields on a torus. ${ }^{1,16,33}$ This algebra also admits central extensions. ${ }^{16,20}$ 


\section{Reduction to the subgroup of volume-preserving diffeomorphisms}

In the case of volume-preserving diffeomorphisms we consider vector fields, further restricted by the condition $\operatorname{div} \mathbf{u}=0$. Let us restrict ourselves to the three-dimensional case $(n=3)$ and let us assume that $\mathbf{m}=(1-\Delta) \mathbf{u}$, so that $\operatorname{div} \mathbf{m}=0$ as well. According to the Helmholtz decomposition theorem for vector fields, $\mathbf{m}$ can be determined only by the quantity $\boldsymbol{\Omega}=\nabla \times \mathbf{m}$. Therefore we can write the Lie-Poisson brackets $(25)$ in terms of $\boldsymbol{\Omega}$. Indeed, one can compute that

$$
\frac{\delta A}{\delta \mathbf{m}}=\nabla \times \frac{\delta A}{\delta \boldsymbol{\Omega}}
$$

Thus

$$
\nabla \cdot \frac{\delta A}{\delta \mathbf{m}}=\nabla \cdot\left(\nabla \times \frac{\delta A}{\delta \boldsymbol{\Omega}}\right)=0,
$$

i.e. the vector fields $\frac{\delta A}{\delta \mathbf{m}}$ are divergence-free. Therefore

$$
m_{i} \frac{\delta A}{\delta m_{k}} \partial_{k} \frac{\delta B}{\delta m_{i}}=\partial_{k}\left(m_{i} \frac{\delta A}{\delta m_{k}} \frac{\delta B}{\delta m_{i}}\right)-\left(\partial_{k} m_{i}\right) \frac{\delta A}{\delta m_{k}} \frac{\delta B}{\delta m_{i}}
$$

and from (25) we obtain

$$
\begin{aligned}
\{A, B\} & =\int\left(\partial_{k} m_{i}\right)\left(\frac{\delta A}{\delta m_{k}} \frac{\delta B}{\delta m_{i}}-\frac{\delta B}{\delta m_{k}} \frac{\delta A}{\delta m_{i}}\right) \mathrm{d}^{3} \mathbf{x} \\
& =\int \boldsymbol{\Omega} \cdot\left(\frac{\delta A}{\delta \mathbf{m}} \times \frac{\delta B}{\delta \mathbf{m}}\right) \mathrm{d}^{3} \mathbf{x} \\
& =\int \boldsymbol{\Omega} \cdot\left(\left(\nabla \times \frac{\delta A}{\delta \boldsymbol{\Omega}}\right) \times\left(\nabla \times \frac{\delta B}{\delta \boldsymbol{\Omega}}\right)\right) \mathrm{d}^{3} \mathbf{x} .
\end{aligned}
$$

This is the well known Poisson bracket used in fluid mechanics. ${ }^{1,17,26,29,31,34}$ The curl of the equation (22) gives the following equation for $\Omega:^{19}$

$$
\boldsymbol{\Omega}_{t}+(\mathbf{u} \cdot \nabla) \boldsymbol{\Omega}-(\boldsymbol{\Omega} \cdot \nabla) \mathbf{u}=0, \quad \boldsymbol{\Omega}=(1-\Delta)(\nabla \times \mathbf{u}) .
$$

Note that $\mathbf{u}$ can be expressed through $\boldsymbol{\Omega}$ :

$$
\mathbf{u}[\boldsymbol{\Omega}]=(1-\Delta)^{-1}\left(\nabla \times \int \frac{\boldsymbol{\Omega}\left(\mathbf{x}^{\prime}\right)}{4 \pi\left|\mathbf{x}-\mathbf{x}^{\prime}\right|} \mathrm{d}^{3} \mathbf{x}^{\prime}\right),
$$

thus

$$
H[\boldsymbol{\Omega}]=\frac{1}{2} \int \mathbf{u}[\boldsymbol{\Omega}] \cdot(1-\Delta) \mathbf{u}[\boldsymbol{\Omega}] \mathrm{d}^{3} \mathbf{x}
$$


The vector $\boldsymbol{\Omega}$ is always perpendicular to $\mathbf{u}$. The further reduction to an equation in $n=2$ dimensions is straightforward. Introducing a (scalar) stream function $\psi\left(x^{1}, x^{2}\right)$ we have

$$
\begin{aligned}
\mathbf{u}\left(x^{1}, x^{2}\right) & =\left(-\partial_{2} \psi, \partial_{1} \psi, 0\right)=\mathbf{e}_{3} \times \nabla \psi, \\
\boldsymbol{\Omega}\left(x^{1}, x^{2}\right) & =(1-\Delta)(\nabla \times \mathbf{u})=(1-\Delta) \Delta \psi \mathbf{e}_{3},
\end{aligned}
$$

where $\mathbf{e}_{3}$ is the unit vector in the direction of $x^{3}$. Since $(\boldsymbol{\Omega} \cdot \nabla) \mathbf{u}=\Omega \partial_{3} \mathbf{u}=0$, (29) leads to the equation

$$
\boldsymbol{\Omega}_{t}+(\mathbf{u} \cdot \nabla) \boldsymbol{\Omega}=0
$$

which produces a scalar equation for the stream function $\psi$ due to (30) and (31), or alternatively for $\Omega \equiv \boldsymbol{\Omega} \cdot \mathbf{e}_{3}$. The Poisson bracket that one can find from $(28)$ is

$$
\begin{aligned}
\{A, B\} & =\int \Omega\left(\partial_{1}\left(\frac{\delta A}{\delta \Omega}\right) \partial_{2}\left(\frac{\delta B}{\delta \Omega}\right)-\partial_{2}\left(\frac{\delta A}{\delta \Omega}\right) \partial_{1}\left(\frac{\delta B}{\delta \Omega}\right)\right) \mathrm{d}^{2} \mathbf{x} \\
& =\int \boldsymbol{\Omega} \cdot\left(\nabla\left(\frac{\delta A}{\delta \Omega}\right) \times \nabla\left(\frac{\delta B}{\delta \Omega}\right)\right) \mathrm{d}^{2} \mathbf{x}
\end{aligned}
$$

and the Hamiltonian

$$
H=\frac{1}{2} \int \nabla \psi \cdot(1-\Delta) \nabla \psi \mathrm{d}^{2} \mathbf{x}
$$

\section{Acknowledgments}

The author is thankful to Prof. A. Constantin, Prof. V. Gerdjikov and Dr G. Grahovski for stimulating discussions. Partial support from INTAS grant No 05-1000008-7883 is acknowledged.

\section{References}

1. V. Arnold and B. Khesin, Topological Methods in Hydrodynamics (Springer Verlag, New York, 1998).

2. R. Beals, D. Sattinger and J. Szmigielski, Inv. Problems 15, L1 (1999).

3. A. Boutet de Monvel and D. Shepelsky, C.R. Math. Acad. Sci. Paris 343, 627 (2006).

4. R. Camassa and D. Holm, Phys. Rev. Lett. 71, 1661 (1993).

5. A. Constantin, Proc. R. Soc. Lond. A457, 953 (2001).

6. A. Constantin and J. Escher, Acta Math. 181, 229 (1998).

7. A. Constantin, V. Gerdjikov and R. Ivanov, Inv. Problems 222197 (2006); nlin.SI/0603019.

8. A. Constantin, V.S. Gerdjikov and R.I. Ivanov, Inv. Problems, 23, 1565 (2007); nlin.SI/0707.2048. 
9. A. Constantin and R. Ivanov, Lett. Math. Phys. 76, 93 (2006).

10. A. Constantin and R. Ivanov, in Topics in Contemporary Differential Geometry, Complex Analysis and Mathematical Physics, eds: S. Dimiev and K. Sekigawa (World Scientific, London, 2007) 33-41; arXiv:0706.3810 [nlin.SI].

11. A. Constantin, B. Kolev, Comment. Math. Helv. 78, 787 (2003).

12. A. Constantin, B. Kolev, J. Nonlinear Sci. 16, 109 (2006).

13. A. Constantin, W. Strauss, Comm. Pure Appl. Math. 53, 603 (2000).

14. A. Constantin, W. Strauss, J. Nonlinear Sci. 12, 415 (2002).

15. A. Fokas and B. Fuchssteiner, Physica D 4, 47 (1981).

16. H. Guo, J. Shen, S. Wang and K. Xu, Chinese Phys. Lett. 6, 53 (1989).

17. D. Holm, Physica D 133, 215 (1999).

18. D. Holm, J. Marsden, in The breadth of symplectic and Poisson geometry, Progr. Math. 232, (Birkhäuser, Boston, MA, 2005).

19. D. Holm, J. Marsden and T. Ratiu, Adv. Math. 137, 1 (1998).

20. J. Hoppe, Phys. Lett B. 215, 706 (1988).

21. R. Ivanov, Phys. Lett. A 345, 235 (2005); nlin.SI/0507005.

22. R. Ivanov, Zeitschrift für Naturforschung 61a, 133 (2006); nlin.SI/0601066.

23. R. Ivanov, Journal of Nonlinear Mathematical Physics 15, supplement 2, 1 (2008).

24. R. Johnson, J. Fluid. Mech. 457, 63 (2002).

25. B. Khesin, G. Misiołek, Adv. Math. 176, 116 (2003).

26. E.A. Kuznetsov and A.V. Mikhailov, Phys. Lett. A 77, 37 (1980).

27. B. Kolev, J. Nonlinear Math. Phys. 11, 480 (2004).

28. J. Lenells, J. Diff. Eq. 217, 393 (2005).

29. D. Lewis, J. Marsden, R. Montgomery and T. Ratiu Physica D 18, 391 (1986).

30. G. Misiołek, J. Geom. Phys. 24, 203 (1998).

31. P.J. Morrison, Rev. Mod. Phys. 70, 467 (1998).

32. E. Reyes, Lett. Math. Phys. 59, 117 (2002).

33. V. Zeitlin, Phys. D 49, 353 (1991).

34. V. Zeitlin, Phys. Lett. A 164, 177 (1992). 